\title{
Inelastical Effects in Classical Field-Theoretical Models with Confinement
}

\author{
YU. A. Simonov \\ ITEP, Moscow, USSR \\ AND \\ J. A. TJon \\ Institute for Theoretical Physics, University of Utrecht, \\ Princetonplein 5, P.O. Box 80006, 3508 TA Utrecht, The Netherlands
}

Received October 24, 1979

Solitons in a class of relativistic field-theoretical models with confinement of spreading waves are considered and their stability is proven under certain conditions for any space dimension. Numerical studies are presented about the collision of solitons and antisolitons in one spatial dimension. In these processes different types of localized structures are generated which are pulsating in time and appear to be stable. The interaction of these new objects, called ss- and sa-breathers, with solitons and antisolitons is also studied.

\section{INTRODUCTION}

Much work has been devoted in the past to a large class of nonlinear evolution equations which are complete integrable and admit soliton solutions [1]. In these models the collision between solitons is purely elastic in the sense that the solitons emerge unmodified from the collision process, suffering essentially only a time delay. On the other hand there exist by now various studies of other nonlinear field-theoretical models indicating that a variety of interesting phenomena can occur in the interaction between solitons [2-7]. In particular, the production of additional localized objects or total disappearance of the original solitons in the collision process between two solitons is possible.

In this paper ${ }^{1}$ we undertake a systematic study of some relativistic models which are closely related to the one considered recently by Werle [8] and Morris [10,11]. It might provide a good field-theoretical description of hadrons with phenomenological properties similar to those of bags [9]. They belong to the class of models not allowing solutions with dissipative character, i.e., solutions behaving like plane

${ }^{1}$ Some of the results have already been published in a letter [7], referred to as $I$. 
or spreading waves at spatial infinity. The above property was first shown by Morris $[10,11]$ and suggests that these models, in the following to be called confining models, have only soliton-like objects as solutions to the classical field equations. Indeed explicit soliton solutions have been found in Ref. [8], but no proof of their stability was given. In a previous example of a confining model, suggested and studied first by Rosen [12] and subsequently by Bialynicki-Birula and Mycielsky [13], it was later on shown by Schick [14] that the relativistic version of this model does not contain stable soliton solutions.

In this paper we undertake a systematic study of certain confining models. First we demonstrate that the solitons in these models are stable in any space dimension provided that certain conditions are imposed on the coupling constants in the Lagrangians. This is done in Section 2 where the models are introduced. Also the asymptotic behaviour of the soliton solutions is discussed. In Section 3 the explicit one-soliton solutions are constructed in one spatial dimension. The linear stability analysis is carried out in detail showing that the only sufficient condition needed for stability of these confining models is the concavity property of the energy dependence on the charge of the solition. In Section 4 we describe the numerical investigations we have carried out on the interaction between solitons in the confining models. In other models [2-6] exhibiting inelastic behaviour in the collision of two solitons either some emission of radiation in the form of an oscillating wave takes place or the production of solitons of the same type is found. However, here we find that the solitons also interact inelastically, but produce one or more additional localized structures of a form different from the original solitons. These objects we call breathers. They are generated in both soliton-soliton and soliton-antisoliton collisions. The common feature of these breathers is their periodic change of shape and size in time. The breathers are similar to the soliton-antisoliton boundstates found in the sine-Gordon equation [1]. It should be noted however that in the soliton-soliton sector of the latter equation they do not exist. In the last section we study the interaction of the produced breathers with solitons. In particular, we find that also these collision processes are always inelastic.

\section{CONFINING Models}

We consider the Lagrangian for a complex scalar field $\psi$ in any space dimension

$$
\mathscr{L}=\left|\partial_{t} \psi\right|^{2}-|\nabla \psi|^{2}-\mathscr{V}\left(|\psi|^{2}\right)
$$

with

$$
\mathscr{V}=\mu^{2}|\psi|^{2}+\lambda|\psi|^{2} b\left(|\psi|^{2}\right)
$$

For confining models $b$ has the properties

$$
b(y \rightarrow 0)=\infty \quad \text { and } \quad y b(y) \rightarrow 0 \quad \text { for } \quad y \rightarrow 0 .
$$


We limit ourselves to those $b(y)$ having in addition the property that $y b(y)$ is positive for $y>0$ and does not have local minima for $y>0$. The solutions of the EulerLagrange equations with the explicit time dependence

$$
\psi(\mathbf{r}, t)=e^{-i \Omega t} \phi(\mathbf{r})
$$

have a non-zero charge

$$
Q=2 \Omega \int|\phi|^{2} d^{n} r
$$

where $\phi$ satisfies the equation

$$
-\Delta \phi+\lambda\left[b\left(|\phi|^{2}\right)+|\phi|^{2} b^{\prime}\left(|\phi|^{2}\right)\right] \phi=\kappa^{2} \phi
$$

with

$$
\kappa^{2}=\Omega^{2}-\mu^{2}
$$

and

$$
b^{\prime}(y)=d b(y) / d y .
$$

It was shown in Ref. [10] that if Eq. (3) holds and $Q \neq 0$, then all solutions of Eq. (6) have a non-dissipative character. We shall now demonstrate that the soliton solutions of Eq. (6) are stable if $b(y)$ satisfies certain conditions. In our considerations a central role is played by the energy functional, given by

$$
E(\{\psi\})=\int\left[\left|\partial_{t} \psi\right|^{2}+|\nabla \psi|^{2}+\mu^{2}|\psi|^{2}+\lambda|\psi|^{2} b\left(|\psi|^{2}\right)\right] d^{n} r
$$

It can easily be seen from Eq. (7) for a system enclosed in a volume $V$, that solutions behaving asymptotically as

$$
\psi \sim\left(\frac{Q}{2 \Omega V}\right)^{1 / 2} e^{i k x-i \Omega t}
$$

contribute an infinite amount of energy to Eq. (7) in the limit $V \rightarrow \infty$ for $b(y)$ satisfying Eq. (3). Therefore all finite energy solutions should be non-dissipative, i.e., localized in space at all times.

The behaviour of the solutions at large $r$ is related to the type of singularity of $b\left(|\psi|^{2}\right)$. Indeed, for

$$
b(y) \sim\left[\ln \ln \frac{1}{y}\right]^{\alpha} \quad \text { for } \quad y \rightarrow 0
$$

we obtain from Eq. (6)

$$
\phi \sim \exp \left[-c r\left(\ln \frac{r}{r_{0}}\right)^{\alpha / 2}\right] \quad \text { for } r \rightarrow \infty .
$$


For more singular $b(y)$, namely

$$
b(y) \sim\left(\ln \frac{1}{y}\right)^{2 \beta /(\beta+2)} \quad \text { for } \quad y \rightarrow 0
$$

we have

$$
\phi \sim \exp \left[-c^{\prime} r^{(\beta+2) / 2}\right] \quad \text { for } \quad r \rightarrow \infty .
$$

In particular, for $\beta=2$ one obtains the model with harmonic oscillator solutions already discussed in Refs. $[3,12,13]$. Note that the asymptotic behaviour given by Eq. (11) is only valid up to a maximal power of two in Eq. (10), which is a critical value. In fact for the interaction

$$
\lambda b(y)=\nu^{2} \ln ^{2} y
$$

we have asymptotically

$$
\phi(r) \sim \exp \left[-\frac{\kappa}{2 \nu} \operatorname{ch}(2 \nu r)\right],
$$

which is completely different from Eq. (11). It is interesting to note, that for any $b(y)$ more singular at $y=0$ than Eq. (12), the solution is exactly zero outside some radius, while at that radius the first derivative is continuous.

Let us now consider some aspects of the stability of our one-soliton solutions. It is instructive first to study the stability in $n$ spatial dimensions of the solution under a scale deformation, which conserves the charge $Q$ [15]. To that end we generate from a given soliton solution $\phi(r) e^{-i \Omega l}$, with $\phi$ real, a family of functions

$$
\phi_{\beta}(r) e^{-i \Omega(\beta) t}=\left(\frac{\Omega}{\Omega(\beta) \beta^{n}}\right)^{1 / 2} \phi\left(\frac{r}{\beta}\right) e^{-i \Omega(\beta) t} .
$$

Substituting this in the energy functional, we obtain

$$
E(\beta)=\frac{\Omega}{\Omega(\beta)}\left\{\left(\mu^{2}+\Omega^{2}(\beta)\right) S+S_{1} \beta^{-2}+\lambda S_{2}(\beta)\right\}
$$

with

$$
\begin{aligned}
S & =\int \phi(r)^{2} d^{n} r, \\
S_{1} & =\int(\nabla \phi(r))^{2} d^{n} r \\
S_{2}(\beta) & =\int \phi(r)^{2} b\left[\frac{\Omega}{\Omega(\beta) \beta^{n}} \phi^{2}(r)\right] d^{n} r .
\end{aligned}
$$

For $\Omega(\beta)$ such that $\Omega(\beta) \beta^{n} \rightarrow \infty$ when $\beta \rightarrow \infty$, we see from Eqs. (16) and (3) the property

$$
S_{2}(\beta) \rightarrow \infty \quad \text { for } \beta \rightarrow \infty .
$$


If $\Omega(\beta) \beta^{n} \rightarrow 0$ for $\beta \rightarrow \infty$, the behaviour of $S_{2}(\beta)$ depends on that of $b(y)$ for large $y$. For stability it is necessary that

$$
b(y)>0 \quad \text { for } \quad y \rightarrow \infty
$$

since otherwise the energy functional $E(\beta)$ becomes unbounded from below if $\Omega(\beta) \beta^{n} \rightarrow 0$ and $\beta \rightarrow \infty$, resulting into spreading of the soliton solution in space with diminishing frequency $\Omega$. Condition (18) is not satisfied by the relativistic model in Refs. $[12,13]$ where $b(y)=\ln \left(y^{-1}\right)$. Our conclusion about the instability of that model is in accordance with Ref. [14]. One may add another term to the interaction to make $b(y)$ satisfy condition (18) as was done in Ref. [11]

$$
b(y)=y+\lambda_{2} \ln y^{-1} .
$$

Linear stability analysis can also be carried out by considering a small perturbation from the original solution $\phi(r) e^{-i \Omega t}$

$$
\psi(r, t)=e^{-i s t}\left[\phi(r)+e^{i \omega t} \chi(r)+e^{-i \omega^{*} t} \chi^{\prime}(r)^{*}\right]
$$

The equation of motion yields

$$
\begin{aligned}
-\Delta \chi_{1}+W(\phi) \chi_{1} & =(\Omega-\omega)^{2} \chi_{1}+2 \omega \Omega\left(\chi_{1}-\chi_{2}\right), \\
-\Delta \chi_{2}+Z(\phi) \chi_{2} & =(\Omega-\omega)^{2} \chi_{2}-2 \omega \Omega\left(\chi_{1}-\chi_{2}\right)
\end{aligned}
$$

with

$$
\chi_{1}=\chi+\chi^{\prime}, \quad \chi_{2}=\chi-\chi^{\prime}
$$

and

$$
\begin{aligned}
& W(\phi)=\lambda\left\{b+5 \phi^{2} b^{\prime}+2 \phi^{4} b^{\prime \prime}\right\}+\mu^{2}, \\
& Z(\phi)=\lambda\left\{b+\phi^{2} b^{\prime}\right\}+\mu^{2} .
\end{aligned}
$$

Translational invariance requires $\Omega^{2}$ to be an $n$-times degenerate eigenvalue of Eq. (20) with $\chi_{2}=0$. A necessary condition for stability of the solution for small perturbations is when all the eigenfrequencies $\omega$ are real. It holds when all the eigenvalues of

$$
\begin{aligned}
& -\Delta \chi_{1}+W(\phi) \chi_{1}=\lambda \chi_{1}, \\
& -\Delta \chi_{2}+Z(\phi) \chi_{2}=\lambda \chi_{2}
\end{aligned}
$$

are non-negative. To ensure this, it is sufficient to require

$$
\begin{gathered}
W(\phi) \geqslant 0, \\
Z(\phi) \geqslant 0 .
\end{gathered}
$$


Presumably, conditions (23) are too strong. Comparing Eq. (22b) with Eq. (6) we see that the original solution $\phi(r)$ is an eigenstate of Eq. (22b) with eigenvalue $\Omega^{2}$. Moreover, since the $s$-wave solutions in general do not have any nodes, this eigenstate has to be the ground state. Consequently all the eigenvalues of Eq. (22b) are greater than zero. The condition of non-negative eigenvalues for Eq. (22a) can be relaxed to allow for the existence of one negative eigenvalue [16]. A sufficient condition for linear stability in that case is given by

$$
\frac{d \Omega}{d Q}<0 .
$$

Using Eqs. (5) and (7) one can readily prove that

$$
\frac{d E}{d Q}=\Omega(Q)
$$

and therefore condition (24) reduces to $d^{2} E / d Q^{2}<0$, i.e., the curve $E(Q)$ should be concave. This condition can easily be understood from the following consideration. Suppose our soliton can decay into two (or more) solitons, then we have in view of the conservation laws of energy and charge

$$
\begin{aligned}
E(Q) & \geqslant E_{1}\left(Q_{1}\right)+E_{2}\left(Q_{2}\right), \\
Q & =Q_{1}+Q_{2} .
\end{aligned}
$$

To forbid these decays one needs precisely the concavity condition.

In the following we consider two specific models of the confining type

$$
\begin{aligned}
& \text { I } \quad \mathscr{V}=\mu^{2}|\psi|^{2}+\lambda|\psi|^{2-\alpha}, \quad \lambda>0, \quad 1>\alpha>0, \\
& \text { II } \mathscr{V}=\mu^{2}|\psi|^{2}+\nu^{2}|\psi|^{2} \ln ^{2}|\psi|^{2} .
\end{aligned}
$$

Both models satisfy the necessary condition for stability given by Eq. (18). With respect to the linear stability criteria the model I satisfies the positivity condition (22) for all $\lambda>0$, while this is true for model II if

$$
\nu^{2}<\mu^{2} / 5
$$

Let us now consider the concavity condition (24). The field equation for model 1 has the form

$$
-\Delta \phi+\lambda\left(1-\frac{\alpha}{2}\right) \phi^{1-\alpha}=\kappa^{2} \phi
$$

with $\kappa^{2}=\Omega^{2}-\mu^{2}$. On substituting

$$
\phi=\left(\frac{\lambda}{\kappa^{2}}\right)^{1 / \alpha} \chi(\kappa r), \quad y=\kappa r
$$


we obtain an equation for $\chi$

$$
-\Delta \chi+\left(1-\frac{\alpha}{2}\right) \chi^{1 \sim \alpha}=\chi
$$

which does not contain $\kappa$ and therefore the charge can be written as

$$
Q=2 \Omega \int \phi^{2} d^{n} r=\frac{2 \Omega}{\kappa^{n}}\left(\frac{\lambda}{\kappa^{2}}\right)^{2 / \alpha} C_{1}
$$

with

$$
C_{1}=\int \chi^{2}(y) d^{n} y .
$$

Using Eq. (34) condition (24) can easily be verified for any number of spatial dimensions $n$. For model II Eq. (24) imposes some conditions on the interaction parameters. The field equation reads in this case

$$
-\Delta \phi+\nu^{2} \phi\left[\ln ^{2}\left(\phi^{2}\right)+2 \ln \left(\phi^{2}\right)\right]=\kappa^{2} \phi .
$$

Restricting ourselves to isotropic solutions, the substitution

$$
\phi=\exp \left[-\frac{\kappa}{v} F(r)\right]
$$

yields

$$
-\frac{F^{\prime 2}}{\nu^{2}}+4 F^{2}+\frac{1}{\kappa}\left[\frac{F^{\prime \prime}}{\nu}+\frac{n-1}{\nu r} F^{\prime}-4 \nu F\right]=1 .
$$

For $n=1$ the solution is simply given by

$$
F=\frac{1}{2} \cosh (2 v x),
$$

while the charge $Q$ is

$$
Q(\omega)=\frac{\omega}{\nu} K_{0}\left(\frac{\kappa}{\nu}\right),
$$

where $K_{0}$ is the modified Besselfunction of zeroth order. From Eq. (38) one finds using the explicit form of $K_{0}(z)$ that $d Q / d \Omega<0$ if the inequality holds

$$
\nu^{2}<21.68 \mu^{2} .
$$

For higher than one dimension we infer from Eq. (36) that for sufficiently large $\kappa / \nu$ we have the same solution for $F$ as that given by Eq. (37). Consequently the condition $d Q / d \Omega<0$ is satisfied for sufficiently small $Q$. Of course in order to establish linear stability for the cases not satisfying Eq. (30) the eigenvalue equation (22a) has to be studied. In the next section we show that in the one-dimensional case there is at most one negative eigenvalue. 
From the above analysis we see that model I has stable soliton solutions for all positive values of $\lambda$ and $\alpha$ in any spatial dimension. The same holds for model II in one spatial dimension provided that Eq. (39) is fulfilled, while in higher dimensions this is true for small enough $v^{2}$.

\section{ONE-SOLiton SOLUTIONS}

In the subsequent sections we confine ourselves to the case of one spatial dimension. We first look for static one-soliton solutions which are of the form

$$
\psi(x, t)=\phi(x) e^{-i \Omega t} .
$$

Taking $\phi(x)$ real, the resulting field equation for $\phi(x)$, Eq. (6), can directly be integrated to yield

$$
\left(\frac{d \phi}{d x}\right)^{2}=\left[\lambda b\left(\phi^{2}\right)+\kappa^{2}\right] \phi^{2}+c_{0}
$$

with $c_{0}$ a constant of integration and $\kappa^{2}=\Omega^{2}-\mu^{2}$. For the two models introduced in the previous section, Eq. (41) can further be integrated with the boundary condition $\phi(x) \rightarrow 0$ for $x \rightarrow \pm \infty$, leading to explicit analytic expressions for the solitons. They are given by

$$
\begin{aligned}
& \phi_{s}(x)=\left(\frac{\lambda}{\kappa^{2}}\right)^{1 / \alpha}\left(\cos \frac{\alpha \kappa x}{2}\right)^{2 / \alpha} \quad \text { for }|x|<\frac{\pi}{\alpha \kappa} \equiv x_{0} \\
& =0 \\
& \text { for }|x|>x_{0}
\end{aligned}
$$

for model I and

$$
\phi_{8}(x)=\exp \left[-\frac{\kappa}{2 \nu} \cosh (2 \nu x)\right]
$$

for model II. Here we have assumed that $\mu^{2}>0$ and $\kappa^{2}>0$. The total charge and energy of these solutions can immediately be calculated from Eqs. (5) and (7). The results for the models I and II are

$$
\text { I } \begin{aligned}
E(\Omega) & =\frac{4}{\alpha \kappa}\left(\frac{\lambda}{\kappa^{2}}\right)^{2 / \alpha}\left[\kappa^{2}+\mu^{2}\left(1-\frac{\alpha}{4}\right)\right] B(\alpha), \\
Q(\Omega) & =\frac{4 \Omega}{\alpha \kappa}\left(\frac{\lambda}{\kappa^{2}}\right)^{2 / \alpha}\left(1-\frac{\alpha}{4}\right) B(\alpha),
\end{aligned}
$$

where

$$
\begin{aligned}
B(\alpha) & =\frac{\Gamma\left(2 / \alpha-\frac{1}{2}\right) \Gamma\left(\frac{1}{2}\right)}{\Gamma(2 / \alpha)} ; \\
\mathrm{II} \quad E(\Omega) & =\frac{2 \Omega^{2}}{\nu} K_{0}\left(\frac{\kappa}{\nu}\right)+2 \kappa K_{1}\left(\frac{\kappa}{\nu}\right), \\
Q(\Omega) & =\frac{\Omega}{\nu} K_{0}\left(\frac{\kappa}{\nu}\right),
\end{aligned}
$$


where $K_{i}(z)$ are the modified Besselfunctions of the $i$ th order. Although no analytic form has been found here for the dispersion relation $E(Q)$, it can be calculated in principle from Eqs. (44-48). The following properties can easily be established:

$$
\begin{aligned}
& \text { I } E(Q) \sim Q^{1-\alpha / 4}, \quad Q \rightarrow 0 \text {, } \\
& E(Q) \sim Q, \quad Q \rightarrow \infty ; \\
& \text { II } E(Q) \sim Q \log \frac{\text { const }}{Q}, \quad Q \rightarrow 0 \text {, } \\
& E(Q) \sim Q, \quad Q \rightarrow \infty .
\end{aligned}
$$

Furthermore as we already discussed in the previous section, one has also $E^{\prime \prime}(Q)<0$ for model I with $\lambda, \alpha>0$ and for model II under the restrictive condition (39). The curve $E(Q)$ has numerically been evaluated for model I with with $\alpha=\frac{1}{2}$ and is shown in Fig. 1. From this we see that over an extended region it is very close to the linear dependence given by Eq. (49) for large $Q$-values.

We now turn to the eigenvalue problem obtained from a linear stability analysis. Expanding $\psi(x, t)$ about the soliton solution gives for model II

$$
-\frac{d^{2} \chi(x)}{d x^{2}}+\nu^{2}\left[\left(\frac{\kappa}{\nu} \cosh 2 \nu x\right)^{2}-\frac{6 \kappa}{\nu} \cosh 2 \nu x+4\right] \chi+\mu^{2} \chi=\epsilon \chi
$$

with $\epsilon=(\omega-\Omega)^{2}$. In general the solutions of Eq. (51) are either even or odd functions of $x$. The lowest three eigenstates can be determined analytically. We have for the lowest even states as eigenfunctions

$$
\begin{aligned}
& \chi_{0}(x)=\exp [-\beta \cosh 2 \nu x]\left[4 \beta+\left(1+\left(16 \beta^{2}+1\right)^{1 / 2}\right) \cosh 2 \nu x\right] \\
& \chi_{2}(x)=\exp [-\beta \cosh 2 \nu x]\left[4 \beta+\left(1-\left(16 \beta^{2}+1\right)^{1 / 2}\right) \cosh 2 \nu x\right]
\end{aligned}
$$

with $\beta=\kappa / 2 \nu$. The corresponding eigenvalues are

$$
\begin{aligned}
& \epsilon_{0}=\mu^{2}+\kappa^{2}-2\left(\nu^{4}+4 \kappa^{2} \nu^{2}\right)^{1 / 2}+2 \nu^{2}, \\
& \epsilon_{2}=\mu^{2}+\kappa^{2}+2\left(\nu^{4}+4 \kappa^{2} \nu^{2}\right)^{1 / 2}+2 \nu^{2} .
\end{aligned}
$$

The lowest odd state can also be found. It is given by

$$
\chi_{1}(x)=\sinh (2 \nu x) \exp [-\beta \cosh 2 \nu x]
$$

with eigenvalue $\epsilon_{1}=\Omega^{2}$. It is the mode which is required by the translational invariance of the system. From Eq. (54) we see that for $\nu^{2} \leqslant 4 \mu^{2} / 9$ there are no negative eigenvalues $\epsilon_{n}$ for all values of $\kappa$, which is compatible with Eq. (30). Moreover for larger $\nu^{2}$-values only at most one negative eigenvalue can occur, given by $\epsilon_{0}$. It should be remarked that the existence of at most one negative eigenvalue could be deduced without explicit solution of the eigenvalue spectrum by noting that the translational 


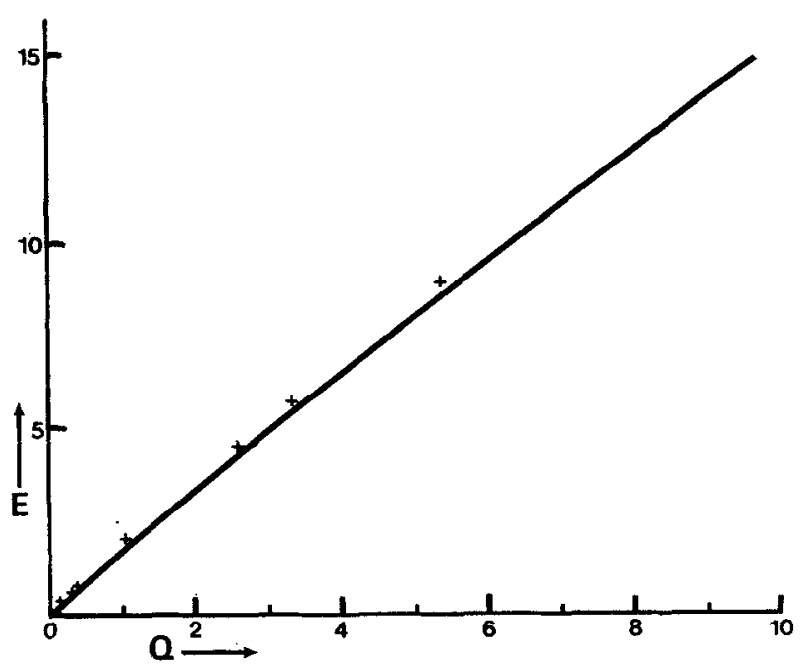

FIG. 1. The energy of the one-soliton solution as a function of the charge $Q$ (solid line). The results from Table II for the ss-breathers are also shown (crusses).

invariant mode (56), to be found from $\phi_{8}$ by infinitesimal translation, has one node, so that only the nodeless groundstate can lie lower than $\Omega^{2}$. Hence the only condition for stability is the concavity of the dispersion relation $E(Q)$, which is true if Eq. (39) holds. This completes our stability analysis of model II.

Having found explicitly the static one-soliton solutions, the solitons moving with a given velocity $u$ can be determined directly by applying a Lorentz transformation. This yields

$$
\psi(x, t)=\exp \left[-i \frac{\Omega}{\left(1-u^{2}\right)^{1 / 2}}(t-u x)\right] \phi_{s}\left(\frac{x-u t-a}{\left(1-u^{2}\right)^{1 / 2}}\right),
$$

where we have assumed that the soliton is centred around $x=a$ at $t=0$.

\section{Soliton-Soliton Collisions}

In this section we describe the results which we have obtained for models I and II in the numerical study of the scattering of two solitons on each other. The initial condition of the system is prepared such that asymptotically when $t \rightarrow-\infty$ the field has the form

$$
\begin{aligned}
\psi(x, t)= & \exp \left[-i \frac{\Omega_{1}}{\left(1-u_{1}^{2}\right)^{1 / 2}}\left(t-u_{1} x\right)\right] \phi_{1}\left(\frac{x-u_{1} t-a_{1}}{\left(1-u_{1}^{2}\right)^{1 / 2}}\right) \\
& +e^{i \theta} \exp \left[-i \frac{\Omega_{2}}{\left(1-u_{2}^{2}\right)^{1 / 2}}\left(t+u_{2} x\right)\right] \phi_{2}\left(\frac{x+u_{2} t-a_{2}}{\left(1-u_{2}^{2}\right)^{1 / 2}}\right),
\end{aligned}
$$


where $\Omega_{i}, u_{i}, a_{i}$ are the frequencies, velocities and displacements of solitons 1 and 2 and $\theta$ is the relative phase. The functions $\phi_{i}$ are the one-soliton solution given by Eqs. (42), (43) for models I and II. We usually take $u_{1}=u_{2}$, since under these circumstances the effects of inelasticity, to be described below, are the strongest. In general, sizable inelastic behaviour is found only for a limited region in the space of parameters of the solitons. Similar findings have been reported for other models [3,5]. Note that for model I the superposition of two solitons in the form (58) is an exact solution when the solitons do not overlap, i.e., the distance between them being greater than $2 x_{0}$. For model II care was taken to have the solitons in the initial state well separated.

The calculations were carried out by letting the solitons move on a line 40 units long with the ends connected to each other. In our units $\mu=1$ and $\Omega$ and $\lambda$ are chosen of the order of one. The $x$-values on the ring were discretized so that in the equation of motion the second derivative in $x$ is simply replaced by $\partial^{2} \psi / \partial x^{2}=\left(\psi_{i+1}-2 \psi_{i}-\right.$ $\left.\psi_{i-1}\right) / \Delta^{2}$. In the actual runs typically 200 meshpoints at equal distance were used so that $\Delta=0.2$. The time integrations were done using the Numerov method with time steps $\Delta t=0.002$. In various runs the number of points in space and time has been changed and checked that the above choice of points is reasonable. As a special testcase the one-soliton expression with $u=0.5, \Omega=1.5$ has been used as initial condition at $t=0$ and verified that up to 400 time units the numerical results were in accordance with the analytic timedependence of the one-soliton solution. In the numerical runs it was explicitly verified that the total energy and charge are conserved up to four decimals. All calculations were performed on a Cyber 73.

First we discuss the results for the model I, which has been studied more extensively. Several cases have been studied in detail.

A. Symmetric collisions.

1. $\Omega_{1}=\Omega_{2}, \theta=0, a_{1}=-a_{2}$ symmetric soliton-soliton collisions,

2. $\Omega_{1}=\Omega_{2}, \theta=\pi, a_{1}=-a_{2}$ antisymmetric soliton-soliton collisions,

3. $\Omega_{1}=-\Omega_{2}, \theta=0, a_{1}=-a_{2}$ symmetric soliton-antisoliton collisions.

B. Non-symmetric collisions.
4. $\Omega_{1} \neq \Omega_{2}, \Omega_{i}>0$, and $\theta=0$ or $\pi$,
5. $\Omega_{1} \cdot \Omega_{1}<0, \theta=0$,
6. $\Omega_{1} \neq \Omega_{2}, \theta \neq 0, \pi$.

Most of our calculations were done for model I with fixed values of $\alpha=\frac{1}{2}, \mu=1$ and $u_{1}=u_{2}=0.5$. In some numerical runs we have changed $u$ to smaller and larger values, but the prononced effects were obtained with $u=0.5$. The values of $\lambda$ and $\Omega$ were taken in the range $0.5 \leqslant \lambda \leqslant 1.5$ and $1.25 \leqslant|\Omega| \leqslant 1.75$. In all cases considered, except for the case 2 , the collision was inelastic. For $\theta \neq \pi$ the solitons are passing each other producing an additional lump of matter and emerging from the 


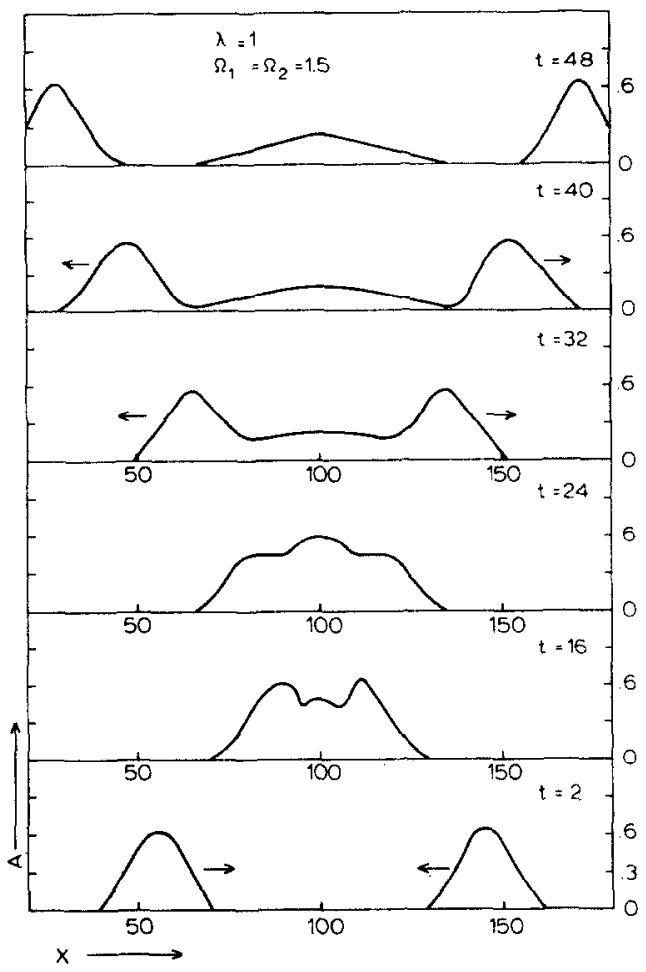

FIG. 2. The amplitude $A$ as a function of $x$ at various times for the soliton-soliton collision process in Model $\mathrm{I}$.

collision process with smaller velocity than before the collision. In Figs. 2 and 3 the time evolution of the amplitude $A=|\psi|$ is shown for the cases 1 and 2 , respectively, with $\Omega_{1}=1.5$ and $\lambda=1$. The following features are found to be common in all the processes (1)-(6) except case 2 .

(i) The solitons experience a time delay during their mutual interaction and which is of the order of several time units.

(ii) A new object is generated in the region of collision which is pulsating periodically in time. The structure can in general be complex as already has been exhibited in I. It does not dissipate in space with time after the complete separation of the outgoing solitons.

Since the qualitative picture resulted also in soliton-antisoliton and non-symmetric soliton-soliton collisions more detailed studies were carried out on these newly created objects, which we call breathers. In particular, those produced in solitonsoliton collisions we will denote as ss-breathers, while sa-breathers are those created in soliton-antisoliton collisions. In these studies the original solitons were removed from the ring after they were well separated from the collision region. The breathers were investigated in regards to the change of their shape in space as a function of time. 


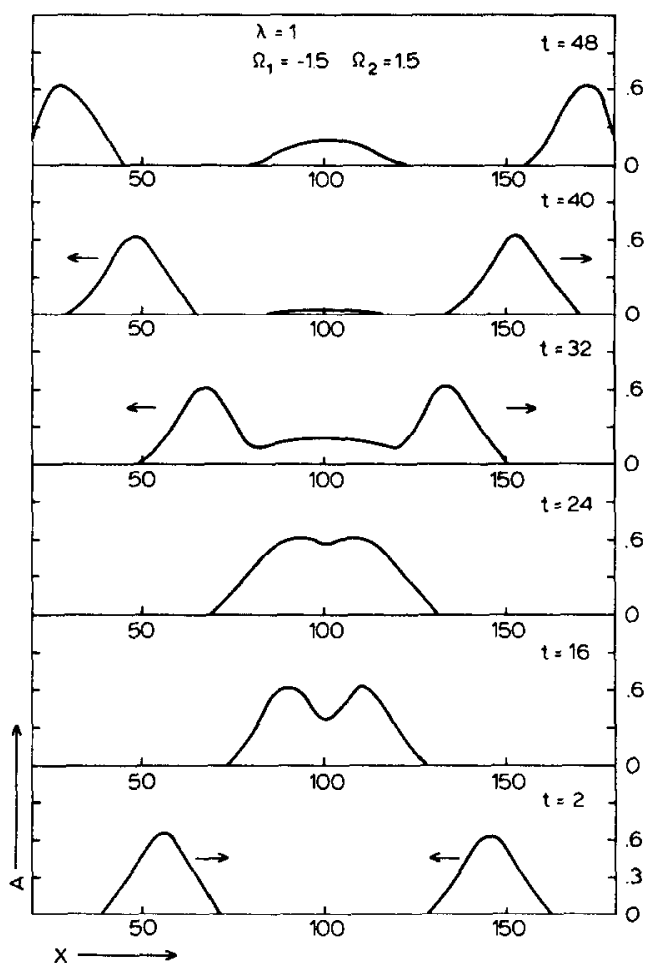

FIG. 3. The same as Fig. 2, but for the antisoliton-soliton collision process.

The amplitude at the center of the ss-breather from Fig. 2 (at $x=100$ in units of $\Delta=0.2$ ) is oscillating with an approximate period of 26 time units, while the ratio between the minimum and maximum is about two. Furthermore the time derivative of the phase at the center is oscillating slightly with the same period around the value of $-\pi / 2$. The shape in $x$ spacc of the ss-breather is clearly changing with time as can be seen in Fig. 4 . The changes in the size of the breather occur periodically with the same period as above $(T \simeq 26)$. In Fig. 4 is also shown the energy density of the ss-breather, behaving in a similar way as the amplitude. For the sa-breather produced in the soliton-antisoliton collision process exhibited in Fig. 3, there exists a period of oscillations which is much smaller, i.e., $T \simeq 2$. Also the time dependence has a more complicated structure as the envelope of the oscillations is pulsating with an additional period of $T \simeq 26$, being incidentally the same as the period found in the ss-breather. As can be seen in Fig. 5 for the amplitude at $x=100$ the period of fast pulsations is slowly changing around the value of $T \simeq 2$. The latter variation corresponds to the periodic change with the slower period of $T \simeq 16$. Also shown in Fig. 5 is the phase of the field at $x=100$, which changes abruptly in time with the period $T \simeq 2$ from $\pi$ to $2 \pi$. In contrast to the case of the ss-breather, the shape of the sabreather can be very complex as can be seen from Fig. 6. In particular, at some times the sa-breather contains various distinct minima and maxima, suggesting that the 

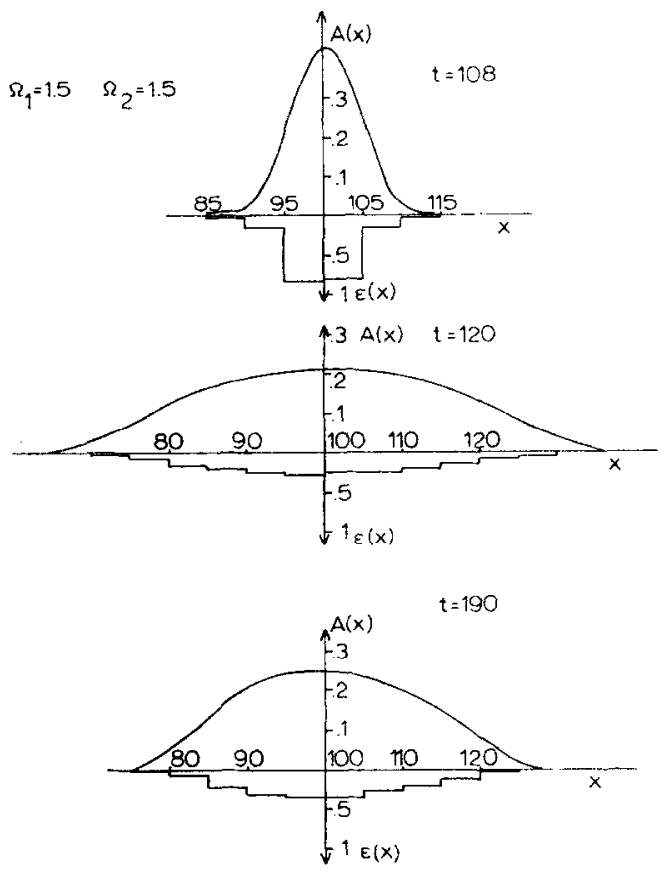

FIG. 4. The amplitude $A$ of the symmetric ss-breather at three characteristic times. The lower curves $\epsilon(x)$ are the corresponding energy density distributions.

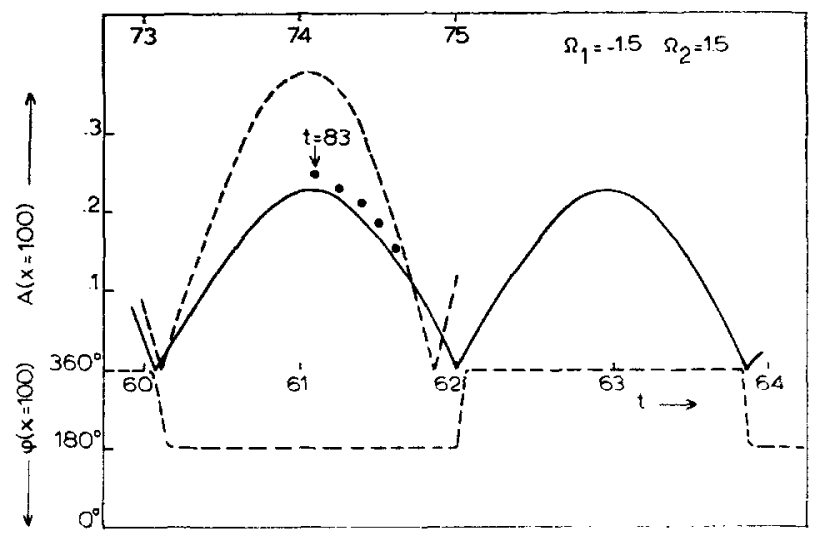

FIG. 5. The amplitude $A$ and the phase $\phi$ at the center of the sa-breather as a function of time $t$. 

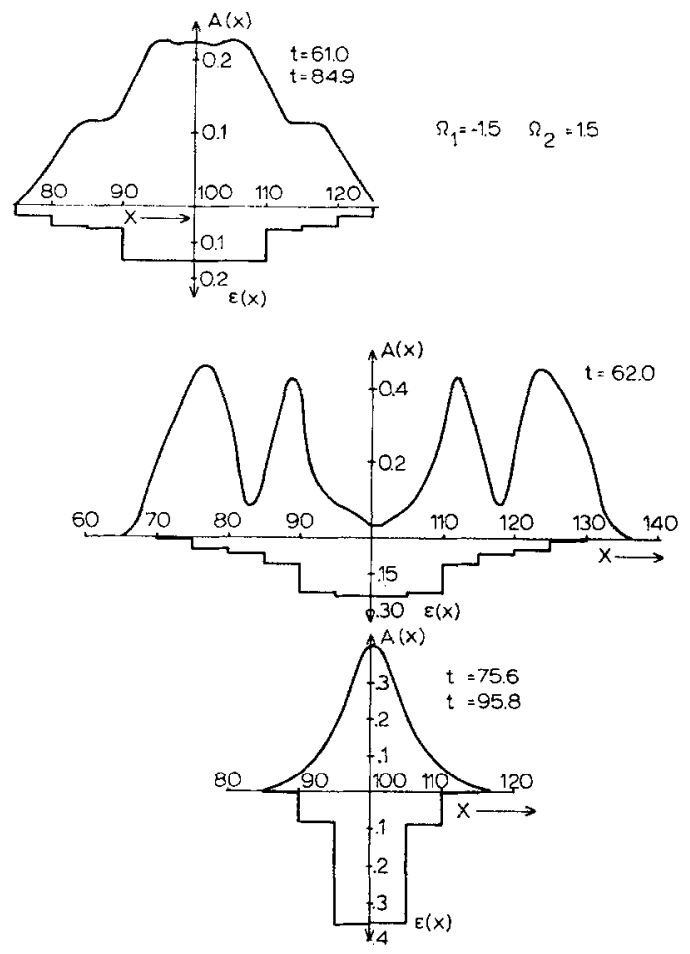

Fic. 6. The same as Fig. 4, but for the symmetric sa-breather.

sa-breather is made out of several lumps of matter bound together and moving relative to each other periodically in time. On the other hand, from Fig. 6 we see that the energy density distribution is comparatively smooth showing no distinct structures.

In contrast to the case of symmetric collisions, where the newly created objects remain centred around $x=100$ because of the symmetry in the initial condition, for non-symmetric collisions they in general move with a certain velocity after the collision. Also non-symmetric soliton-soliton collisions lead to the new feature of the possible production of more than one breather as is shown in Fig. 7 in the $x-t$ plane for the case of $\Omega_{1}=1.25$ and $\Omega_{2}=1.75$. These breathers pulsate periodically in time but asymmetrically in space, so that the position of the maximum in the amplitude is oscillatory around some midpoint in the breather. We consider this property rather as an indication because the numerical accuracy in our calculation is probably not high enough to be conclusive.

Unlike all other cases the antisymmetric soliton-soliton collision behaves elastically. This is shown in Fig. 8 for the case $\Omega_{1}=\Omega_{2}=1.5$. During the first stage of the collision between the solitons they appear separately to be compressed together at the same time developing a tail at the outer ends. At a later stage they change sign in the velocity while gradually recovering their original shape. In that process the tail is 


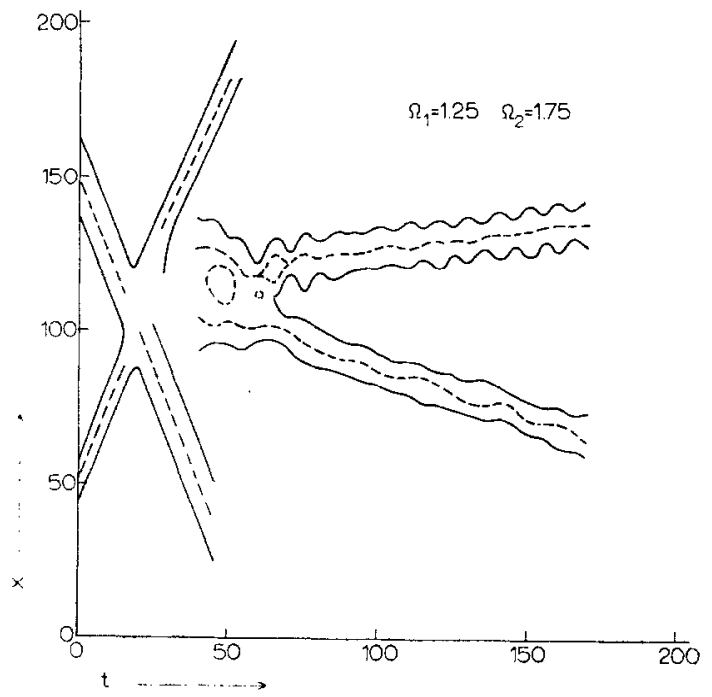

Fig. 7. The time dependence of the asymmetric soliton-soliton collision process with two localized objects being produced. The local maxima of the amplitude are denoted by dashed lines, while the nearby solid lines indicate where the amplitude has decreased by a factor of 2 .

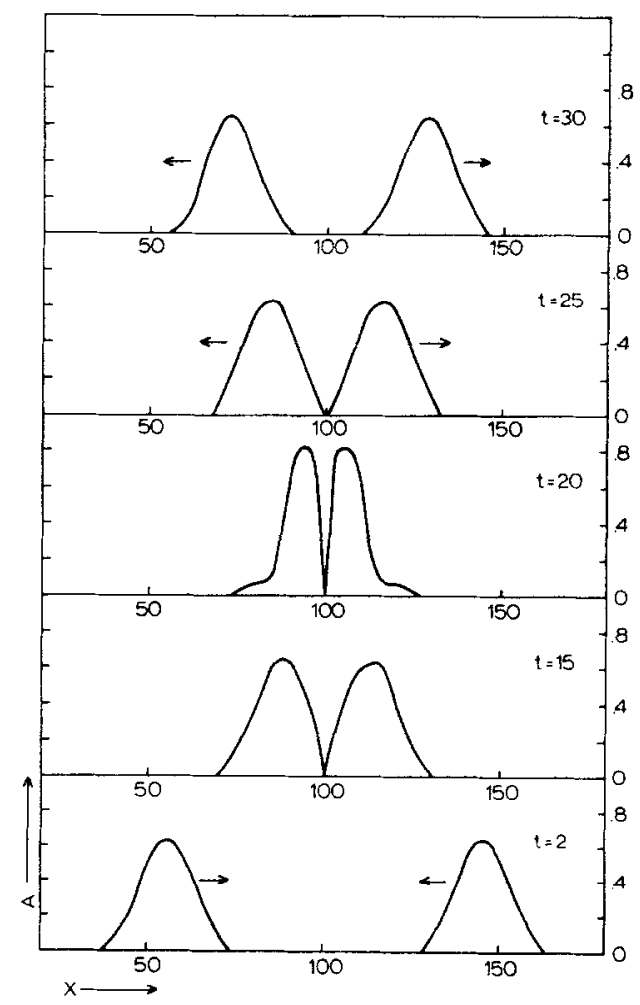

FIG. 8. The same as Fig. 2, but for the antisymmetric soliton-soliton collision process. 


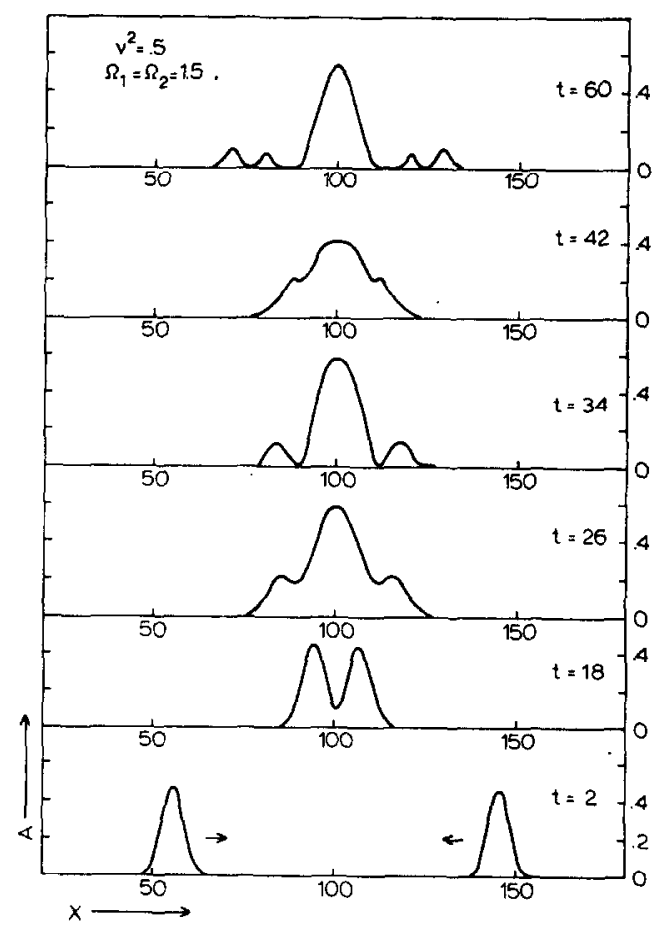

FIG. 9. The same as Fig. 2, but for Model II.

being reabsorbed by the reflected objects. We have checked by running case 6 that for $\Omega_{1}=\Omega_{2}$, that $\theta=\pi$ is a unique initial state with the properties described above. In all other cases breathers are generated.

We also studied numerically model II. A typical example is shown in Fig. 9 for the symmetric soliton-soliton collision process with $\Omega_{1}=1.5$ and $\nu^{2}=0.5$. As can be seen from this figure the time that the solitons interact is relatively long, while various localized structures are produced. Similarly as in model I the amplitude of the object in the interaction region around $x=100$ can vary by a factor of 2 as a function of time. The changes, however, are faster here and more erratic. The main difference between the two models is the high inelasticity found here. Taking as a measure for the elasticity the fraction of total charge lost by the original solitons to the structure in the middle, according to Table I it is for sufficient large $v^{2}$ significantly higher than the $15 \%$ inelasticity found in model I. Also there is a strong dependence on the coupling constant $\nu^{2}$. A very sharp rise in the inelasticity occurs at around $\nu^{2}=0.4$. The time delay suffered from the collision crucially depends on $\nu^{2}$ and is rapidly increasing at $\nu^{2} \approx 1$. For $\nu^{2} \approx 2$ the solitons appear to have fused completely so that no solitons scem to cmerge from the collision process even for times of the order of 200 . 
TABLE I

The Energy $E_{\mathrm{br}}$ and Charge $Q_{\mathrm{br}}$ of the SS-Breather of Model II ${ }^{a}$

\begin{tabular}{ccclll}
\hline$\nu^{2}$ & $F_{\text {tot }}$ & $Q_{\text {tot }}$ & \multicolumn{1}{c}{$E_{\mathrm{br}}$} & \multicolumn{1}{c}{$Q_{\text {br }}$} & \multicolumn{1}{c}{$Q_{\text {br }} / Q_{\text {tot }}$} \\
\hline 0.25 & 2.30 & 1.03 & $5.10^{-3}$ & $\sim 0$ & \\
0.35 & 3.12 & 1.32 & $1.910^{-2}$ & $1.10^{-3}$ & $\sim 10^{-3}$ \\
0.40 & 3.47 & 1.44 & 2.43 & 1.24 & 0.86 \\
0.50 & 4.09 & 1.63 & 2.70 & 1.41 & 0.87 \\
0.75 & 5.30 & 1.95 & 3.60 & 1.77 & 0.91 \\
1.00 & 6.22 & 2.14 & 4.53 & 2.12 & 0.99 \\
\hline
\end{tabular}

${ }^{a} \nu^{2}$ is the coupling constant. $E_{\text {tot }}$ is the total energy and $Q_{\text {tot }}$ the total charge of two colliding solitons in the initial state.

\section{Other Properties of the Breathers}

In the previous section we have described the existence of some localized structures which are produced in the collision between solitons. One of the important and interesting questions is the problem of the stability of these objects. Numerically we have studied the stability properties of the ss- and sa-breathers. The first one was followed on the computer for 1000 time units. The length or period of the pulsations did not change within the accuracy of our computations. The sa-breather was studied up to times of the order of 200, in which period about 100 internal pulsations took place in the object. Also in this case the structure appears to be stable.

One may also determine the charge and energy of these breathers. The results are presented in Table II for the ss-breathers generated in symmetric soliton-soliton collisions for various values of $\lambda$ and $\Omega$. Also, the fraction of the energy and charge transmitted to the breathers can be calculated. They are shown in the two last columns in Table II and are always about 0.14 . Using the energies of the ss-breather from this table we see that if we compare them with the energy of one soliton at rest with the same $Q$ they are about $5 \%$ higher. This is shown in Fig. 1, where the results from Table II are given by crosses, indicating that the breathers may be unstable. We have tried to understand the stability of the ss-breather found in the actual calculation by considering the possibility of the decay of such a ss-breather into two equal solitons with charge $Q / 2$, being in view of symmetry the most probable decay mode. The difference in energy between these two solitons at rest and the ss-breather is also given in Table II in the last but two columns. As is seen it is in general very small. In the reliable cases it is $10^{-3}$ of the mass of the ss-breather and positive, suggesting that if the ssbreather consisted of two equal solitons it would be a bound state or in some cases a resonance with a very small excitation energy. Of course, the additional $\mathrm{cm}$ motion of the solitons needed to separate the two solitons from each other would make the decay process even more prohibitive. 
TABLE II

The Characteristics of the SS-Breather as Generated in the Collision Process of Two Solitons of Model I ${ }^{a}$

\begin{tabular}{lcccccccc}
\hline$\lambda$ & $\Omega$ & $E_{\mathrm{t}}$ & $Q_{\mathrm{t}}$ & $E_{\mathrm{br}}$ & $Q_{\mathrm{br}}$ & $\epsilon_{\mathrm{b}}$ & $E_{\mathrm{br}} / E_{\mathrm{t}}$ & $Q_{\mathrm{br}} / Q_{\mathrm{t}}$ \\
\hline 0.5 & 1.5 & 1.08 & 0.47 & 0.16 & 0.07 & $-1.10^{-8}$ & 0.152 & 0.140 \\
0.75 & 1.5 & 4.79 & 2.39 & 0.71 & 0.34 & $-8.10^{-8}$ & 0.149 & 0.141 \\
1 & 1.4 & 40.0 & 23.1 & 5.7 & 3.3 & $-1.10^{-2}$ & 0.143 & 0.144 \\
1 & 1.5 & 14.1 & 7.56 & 2.0 & 1.1 & 0.014 & 0.142 & 0.140 \\
1 & 1.6 & 5.96 & 2.97 & 0.82 & 0.40 & $7.10^{-3}$ & 0.138 & 0.135 \\
1 & 1.7 & 2.86 & 1.33 & 0.37 & 0.17 & $1.10^{-2}$ & 0.129 & 0.128 \\
1.25 & 1.5 & 32.7 & 18.4 & 4.5 & 2.6 & 0.07 & 0.140 & 0.140 \\
1.5 & 1.5 & 66.2 & 38.24 & 8.9 & 5.4 & 0.04 & 0.135 & 0.140 \\
\hline
\end{tabular}

a The energy of the ss-breather $E_{\mathrm{br}}$ and charge $Q_{\mathrm{br}}$ are given for several values of coupling constant $\lambda$ and frequency $\Omega$ together with the total energy and charge $E_{t}, Q_{t}$ of the two colliding solitons in the initial state. The value $E_{\mathrm{b}}$ is the binding energy of the breather with respect to the two masses of the assumed constituent solitons with charge $Q_{\mathrm{br}} / 2$.

Finally the interaction between breathers and solitons may be of some interest. For this purpose we have studied in some detail processes where a (anti)soliton makes a collision with a breather. The breathers are constructed starting from the symmetric collisions described in the previous section with $\Omega_{1}=1.5$ and $u_{1}=0.5$. At time $t=55$ when the outgoing solitons are well separated these solitons are removed and a leftward moving (anti)soliton is inserted at $x=150$. In all the collision processes condered there were inelastic effects. In Fig. 10 is shown the collision of a ss-breather and a soliton with $\Omega=1.5$ and $u=-0.5$. The soliton passes the ss-breather; at the same time a structure is formed in the tail of the soliton which separates and moves slowly to the right. There is again in the final state a ss-breather moving also to the right with a period of pulsation of $T=22$, being shorter as compared to the period of $T=26$ before the collision. The variations of the amplitude of the newly created object are much smaller than those of the ss-breather, suggesting that within the numerical accuracy it may be a soliton. Similarly letting a soliton collide on a ssbreather appears to produce an additional localized structure, the amplitude of which being almost constant. On the other hand if we consider the collision between an antisoliton on a ss-breather an object is produced with a rapidly varying amplitude indicating that it might be of a sa-breather type. The collision process is shown in Fig. 11. In order to see the possible nature of the localized structures present after the collision, the amplitudes at the maximum of the three structures are plotted in Fig. 12 as a function of time. From this we indeed see the rapid variations in the newly created object.

To summarize, in this paper we have considered a class of field-theoretical models with non-polynomial Lagrangians which admits soliton solutions and gives rise to 


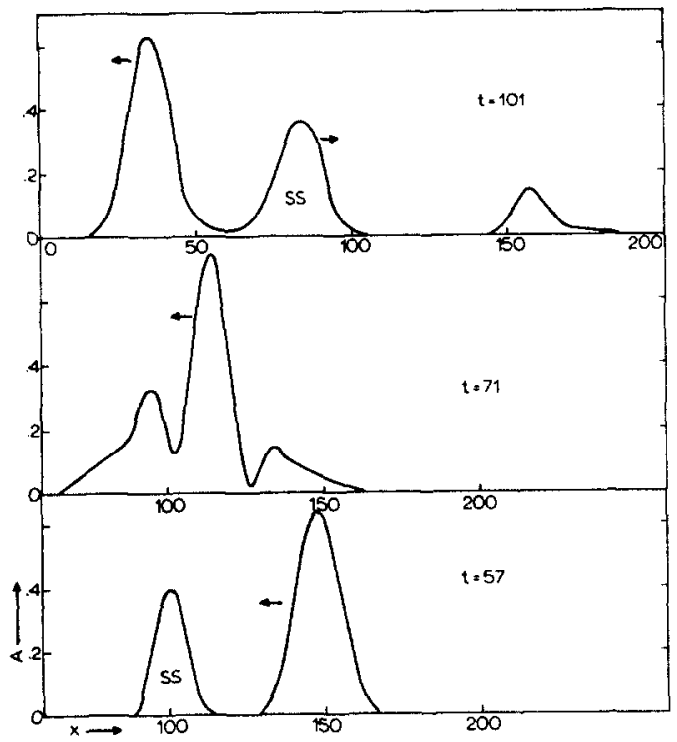

Fig. 10. Three time slices for the collision process of one soliton with a ss-breather showing the situations before, during and after the collision.

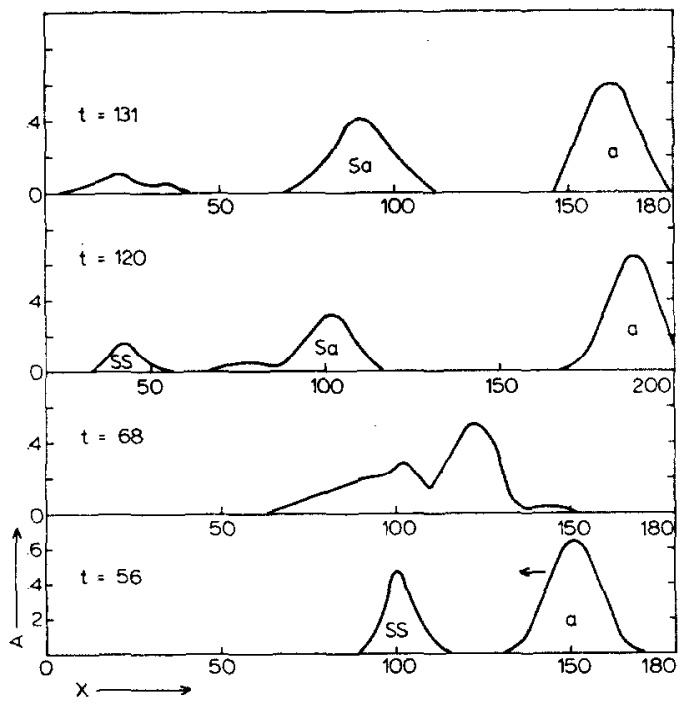

FIG. 11. Four time slices for the collision process of one antisoliton with a ss-breather. 


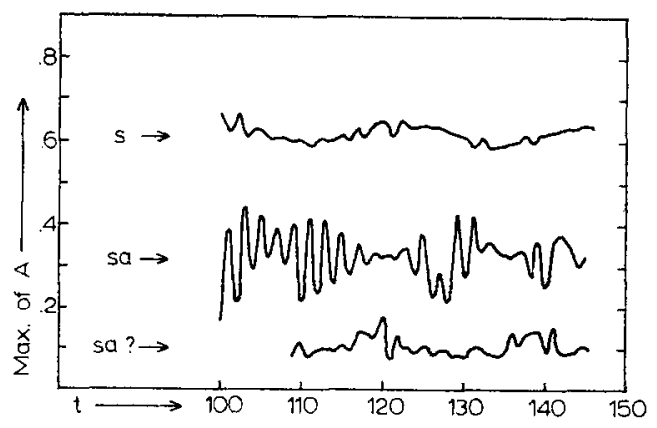

FIG. 12. The time dependence of the maximal amplitudes of the three localized objects in the final state of the collision process of Fig. 11.

remarkable inelastic effects in the collision process between solitons. From our study we have seen that the one-soliton solutions in these models are also stable in higher dimensions. Although we expect that inealstic effects are also present in this case, it would be interesting to see what kind of localized structures are generated.

\section{ACKNOWLEDGMENTS}

This work was done during the periods when one of us (Yu.S.) was visiting the Free University of Amsterdam and the other one (J.T.) the Institute for Theoretical and Experimental Physics in Moscow within the framework of an agreement between the Atomic Commission of the USSR and the Reactor Centre of the Netherlands. The authors would like to thank both Institutes for the hospitality extended to them.

\section{REFERENCES}

1. See, for example, A. F. Scott, F. Y. F. Chu, ANd D. W. Laughlin, Proc, I.E.E.E. 61 (1973), 1443.

2. V. G. Makhankov, Phys. Rep. C 35 (1978), 1.

3. J. Oficjalski and I. Bialynicki-Biruta, Acta Phys. Pol. B 9 (1978), 759.

4. A. R. Santarelli, Nuovo Cimento B 46 (1978), 179.

5. J. C. Lewis and J. A. Tuon, Phys. Lett. A 73 (1979), 275.

6. V. E. GRISHIN et al., Dubna preprint P2-12359, 1979. [in Russian]

7. Yu. A. Simonov and J. A. Tjon, Phys. Lett. B 85 (1979), 380.

8. J. WerLe, Phys. Lett. B 71 (1977), 357; 71 (1977), 367.

9. A. Chodos et al., Phys. Rev. D 9 (1974), 3471.

10. T. F. MORRIS, Phys. Lett. B 76 (1978), 337; 78 (1978), 87.

11. T. F. MORRIS, Canad. J. Phys. 56 (1978), 1405.

12. J. Rosen, Phys. Rev. 183 (1969), 1186.

13. I. Bialynicki-Birula and J. Mycielsky, Ann. Phys. (N.Y.) 100 (1976), 2.

14. P. Schick, Nucl. Phys. B 104 (1976), 137.

15. Yu. A. Simonov, Yad. Fiz. 30 (1979), 1148, 1457.

16. R. Friedberg, T. D. Lee, ANd A. Sirlin, Phys. Rev. D 13 (1976), 2739. 